\title{
Vismarkmap - A Web Search Visualization Technique through Visual Bookmarking Approach with Mind Map Method
}

\author{
Abdullah Al-Mamun \\ Department of Computer Science \& Engineering \\ Daffodil International University \\ Dhaka, Bangladesh
}

\author{
Sheak Rashed Haider Noori \\ Department of Computer Science \& Engineering \\ Daffodil International University \\ Dhaka, Bangladesh
}

\begin{abstract}
Due to the massive growth of information over the Internet, Bookmarking becomes the most popular technique to keep track of the websites with the expectation of finding out the previously searched websites easily whenever are needed. However, present browser bookmark systems or different online social bookmarking websites actually do not let the users to manage their desired searches with appropriate method, so that the users could easily recognize or recall the previously searched websites and its content with the bookmark whenever they are in need. In this paper, a new approach of bookmarking technique has been proposed which will let the users to organize their bookmarks with some features using mind map, a scientifically approved mental model that will help the users to recall easily the previously searched websites' information from the bookmarks and will minimize the tendency to revisit or research the website using the search engines. Basically, the proposed system is more than a mind map as it provides more flexibility to organize the bookmarks.
\end{abstract}

Keywords—hci; visual bookmark; information retrieval; mind map; visualization

\section{INTRODUCTION}

Since the World Wide Web releases to the public, Internet continues to grow and becomes a very important source of information. For seeking information on the web users follow several different strategies such as direct navigation, navigation within a directory and navigation using a search engine; among which the search engines make the information searching process more easier [10] than others.

However, in the user perspective, a very crucial problem is being provided very less care, which is about coming back to information they have previously found [5]. During a particular search session a search engine might find the desired search results; though, finding the same result users might require to search or navigate again with extensive effort, since due to the rapid increment of the registered domain (approximately 350 million) since last decade [13], the World Wide Web is becoming massive and largely disorganized. Therefore, finding out a previously visited webpage might lead to an unsatisfying and unproductive experience. In addition, different search engines provide the search results of different web sites in list based approach. However, users prefer to devote very small amount of time for the navigation inside web sites, rather than using the search results list they prefer to jump from one site to another [10]. As the quality of information on the Web varies and the user has to make a judgment within very short period of time before jumping from one site to another, users must need to have a quick visualization model in which they will be able to compare among the similar or dissimilar search results to find out their expected web site very quickly, which might provide them a satisfying information searching experience as they will need to put less mental effort.

On the other hand, to facilitate the process of finding previously searched results, present commercial web browsers such as Google Chrome, Mozilla Firefox and Microsoft Internet Explorer are providing almost identical functions for returning to these pages, such as Back, the history list, and bookmarks. The aforementioned mechanisms should greatly be used, as according to the past research [11] a person browses mostly (60\% of all the pages) previously visited pages. However, due to several problems with these mechanisms, bookmark and history systems are rarely used $[11,12]$. One of the main reasons is the scattered and unintegrated method of the browsers re-visitation systems [5]. The major functions such as Back, history and bookmarks, all use different models, user interfaces, and provides numerous techniques of organizing and visualizing groups of candidate pages. As a result, recalling a website from the history list becomes difficult.

To overcome the aforementioned problems, in this paper, a new bookmarking technique has been proposed which will let the users to organize their bookmarks using mind map, which is a scientifically approved mental model. With the help of the mind maps, it will help to find back easily their previously searched websites by minimizing their (users) dependency on the search engines, which will provide a better searching experience.

The rest of the paper is organized as follows. Some of the important related researches are reported in section 2. In section 3, the solution against the traditional bookmarking systems is proposed. The proposed solution is explained in details in section 4. In Section 5 and 6, the evaluation of the experiment and the related discussion is presented respectively. 


\section{RELATED RESEARCH}

At present, for the bookmarking process user use either renowned web browsers' default bookmark system or different social bookmarking websites, such as delicious, Pinterest and Diigo etc. In present commercial web browsers such as Google Chrome, Mozilla Firefox and Microsoft Internet Explorer, we bookmark our favorite websites with tags. The Delicious allows users to bookmark the useful searches with tags and to share with people. Unfortunately from the researches [3] it has been found there is a natural convergence of the tags on the Delicious website. Even so, these tags lack embedded semantics, so when observing Delicious as tagging platform, the common ambiguity problems of tagging (such as synonyms or false tags) [4] are encountered. In Diigo ${ }^{1}$ users can create bookmark list of web sites along with tags and also are able to keep notes with specific fragments of a webpage which are visible when those websites are revisited. With the Pinterest users are able to bookmark the searches along with thumbnail preview, tags and personal notes. Moreover, in past, several applications were implemented to organize and visualize the web search results. Shaun Kaasten and Saul Greenberg [5] proposed an integrated system which worked in Microsoft Internet Explorer. Basically they designed a new view of history list to represent the visited pages with visual thumbnails along with titles and respective URLs. It includes two types of bookmarking process such as Implicit and Explicit bookmarking with the help of Dog Ear metaphor. The most visited pages are marked automatically with Implicit bookmark and Explicit bookmarking is done by the users. In addition, it supports searching the bookmarks based upon the most visiting frequency. David Gotz proposed a system [9] where user can drag and drop URL of a link from browser to bookmark and can create a hierarchical tree of connected nodes of URLs. Besides, Users can store full webpage or a particular segment of a webpage as a node in the tree.

After analyzing the aforementioned works, it can be concluded that most of the researches followed either simple data clustering or symbolic representation or spatial representation with the help of tree to organize the bookmarks. However, these approaches are not appropriate enough to visualize information and knowledge of the bookmarks in coherent approach as those are not following the appropriate cognitive theory.

\section{PROPOSED SOLUTION}

As user has to decide very quickly before jumping from one bookmarked site to another by comparing among the similar or dissimilar bookmarks very quickly, in the proposed solution the focus is put on the visualization of the information and knowledge of the bookmarked websites following the cognitive fit theory [1]. Proper visualization mitigates the limitation of the working memory and helps the learner overcome problems during the process of learning and problem solving. According to Sweller and Chandler [14] visualizations may reduce cognitive load and in [15], [16], and [17], it is mentioned that visualization expand the capability of an individual's memory for coping with complex cognitive task requirements. Cox [15] and Scaife \& Rogers [18] mentioned another significant reason; visualizations can enhance our processing ability by visualizing abstract relationships between visualized elements and may serve as a basis for externalized cognition.

To visualize information and knowledge together, in the proposed solution Synergistic approach is followed. The Synergistic approach aims at integrating knowledge and information visualization in coherent approach [8]. It is claimed that map based approach such as concept map can be used for mapping and managing conceptual knowledge among information [6]. According to cognitive fit theory [1], it is also known that, graphs work as representations of the spatial problems because they present spatially related information which emphasizes relationships in the data. Even though, concept map is helpful for organizing knowledge, it is not considered as complete mental model as it requires imagerybased elements to comprehensively represent the knowledge [2]. In addition, research [5] shows organizing bookmarks with thumbnail images, makes the process of scanning the bookmark list easy for the users. Therefore, in this paper a new bookmarking technique VisMarkMap is proposed with the help of mind map as it is very effective way of the Synergistic approach to represent information and knowledge coherently and removes the shortcomings of the concept maps; because, with the help of mind map [Figure 3] one can represent the similarity or dissimilarity among the bookmarks along with visual imagery elements and tags. In addition, the proposed technique also supports the visualization of the quality of the bookmarks based upon the most visiting frequency and the five star rating, provided by the users though ranking mechanism. On the other hand, discrete values cannot be presented directly with graphs such as mind maps or concept maps [1]. For symbolic problem representations one can use tables or list based approach because they present symbolic information and emphasize discrete data values. Therefore, for visualizing all mind maps' bookmarks together, a tag list based approach [Figure 8] has been chosen to give the users an overall knowledge about all the bookmarks of all mind maps.

\footnotetext{
${ }^{1}$ https://www.diigo.com/
} 


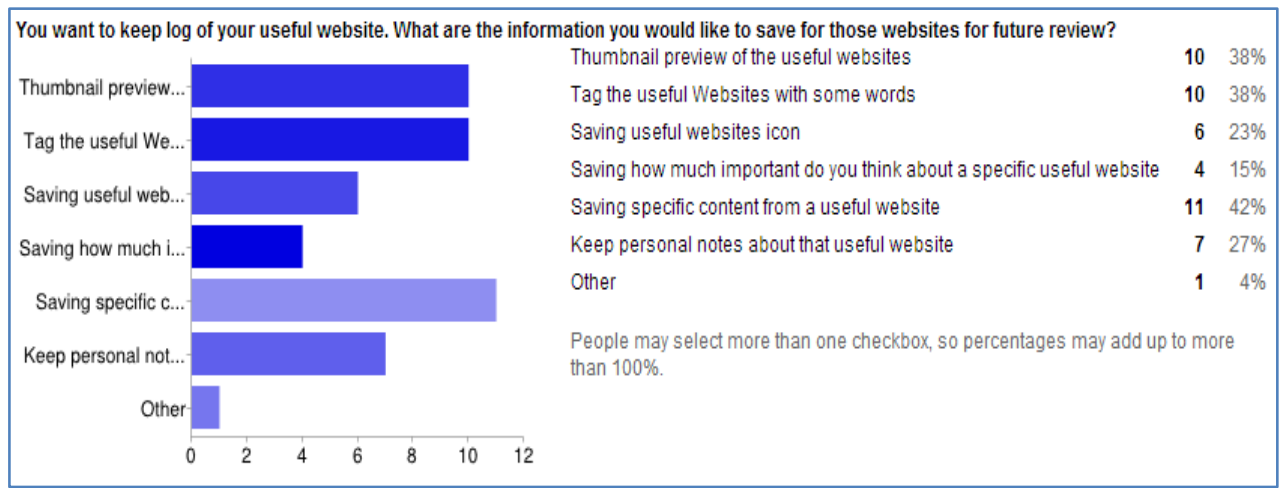

Fig. 1. Initial survey result for bookmark components

\section{VISMARKMAP-VISUAL BOOKMARKING WITH MIND MAP METHOD}

At the beginning a survey has been conducted in order to find out the expected information to be visualized about a website bookmark, in which 26 people (students and teachers of different college and universities) participated. From the outcome of the survey [Figure 1] it is found that most users expect to see the thumbnail, the associated tag and specific content kept from that respective web site. Besides, users also wanted to keep personal notes about the bookmarked web sites. Therefore, the proposed visual bookmarking system (VisMarkMap), which is implemented as a browser extension for Chrome, allows users to organize their bookmarks along with mind maps with above mentioned features. The overall process of the VisMarkMap is depicted in Figure 2.

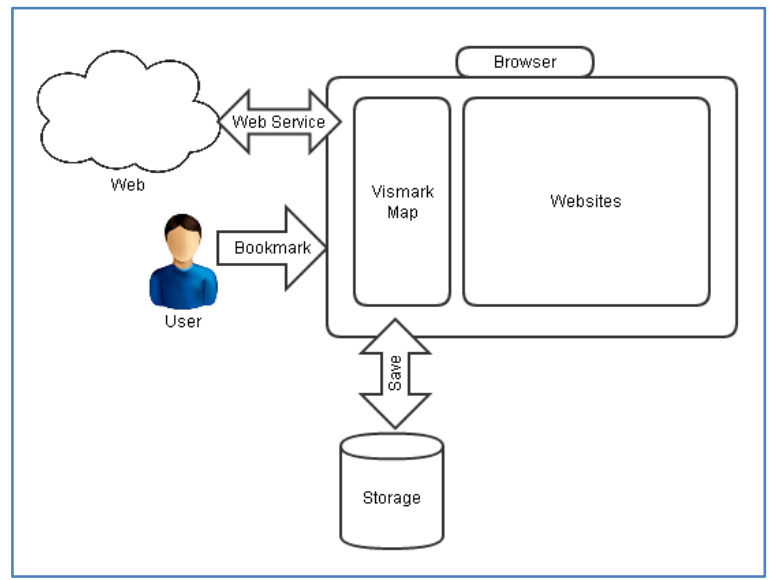

Fig. 2. VisMarkMap architecture

During a search session, user can interact with the visual bookmark button on the browser and the bookmark panel will then be shown on the left side of the browser [Figure 9]. Then a user can start creating new mind map with NewMap button and create new node with NewNode button. After that, the user will select the desired URL (website or image or video) from the browser address bar and will perform a simple drag \& drop operation to fetch the URL on the bookmark panel [Figure 3]. The VisMarkMap will then utilize a web service to create the necessary thumbnail and big preview image or small preview clip for video bookmark associated with the bookmarked URL.

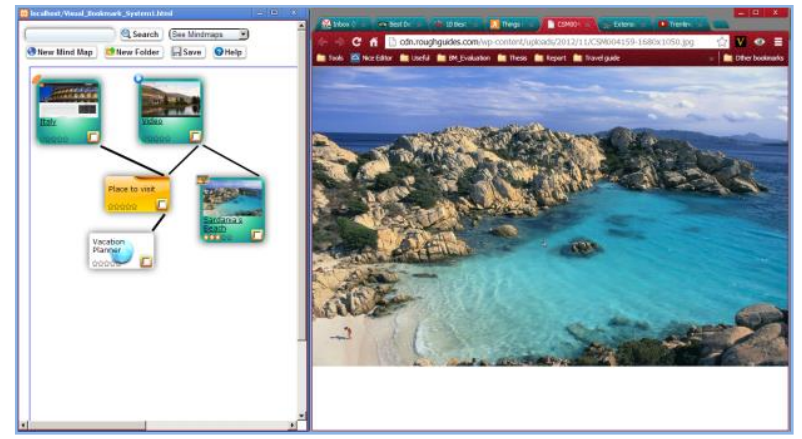

Fig. 3. Bookmarking in mind map

In addition, Figure 4, Figure 5 and Figure 6 show a user can select the desired text from bookmarked website and can keep as a note along with the big preview image, which mitigates the necessity of visiting the respective website again. This system will also assist users to keep date and to perform necessary tagging on the bookmarks as well as to create groups by allowing making desired relationships among the similar bookmarks to form a mind map.

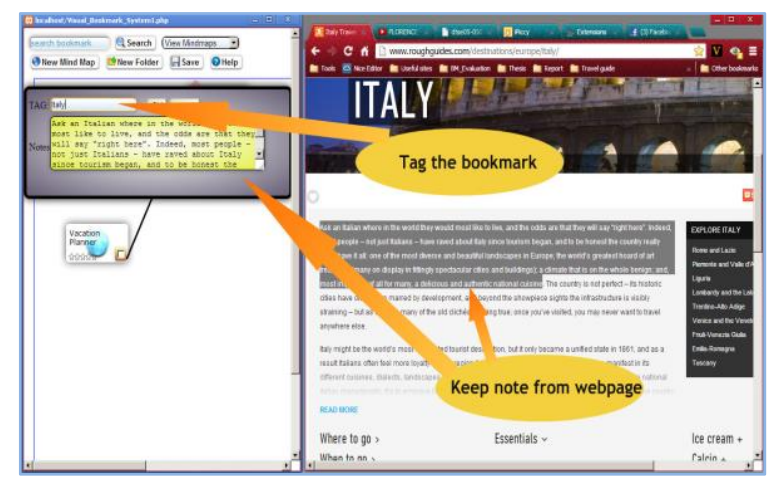

Fig. 4. Tagging and keeping notes

There is also a five star rating mechanism with which users can mark the importance rate of the bookmarks in a mind map [Figure 7]. Besides, based on the most visiting frequency the bookmarks will be highlighted on the mind map [Figure 7]. The summary of the mind maps will also be shown as tag 
boxes on the home panel [Figure 8], which contain the user defined tags of the bookmarked sites.

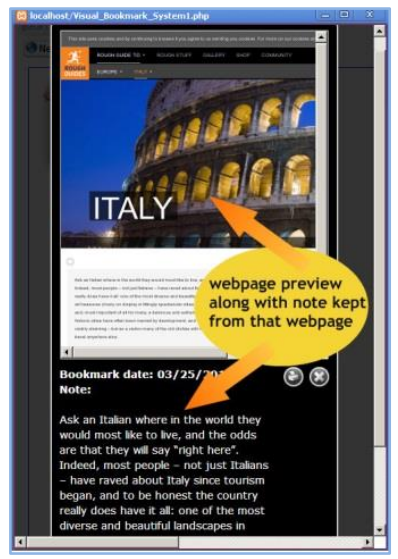

Fig. 5. Preview of a bookmarked website

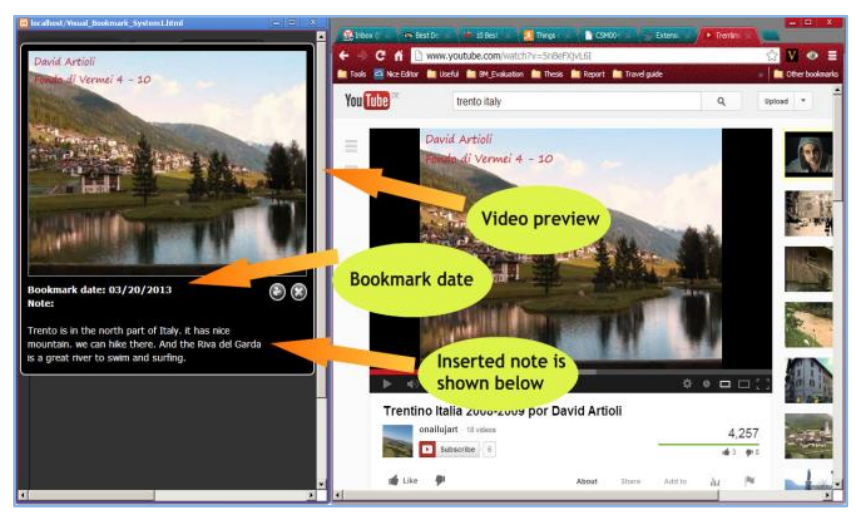

Fig. 6. Preview of a bookmarked video

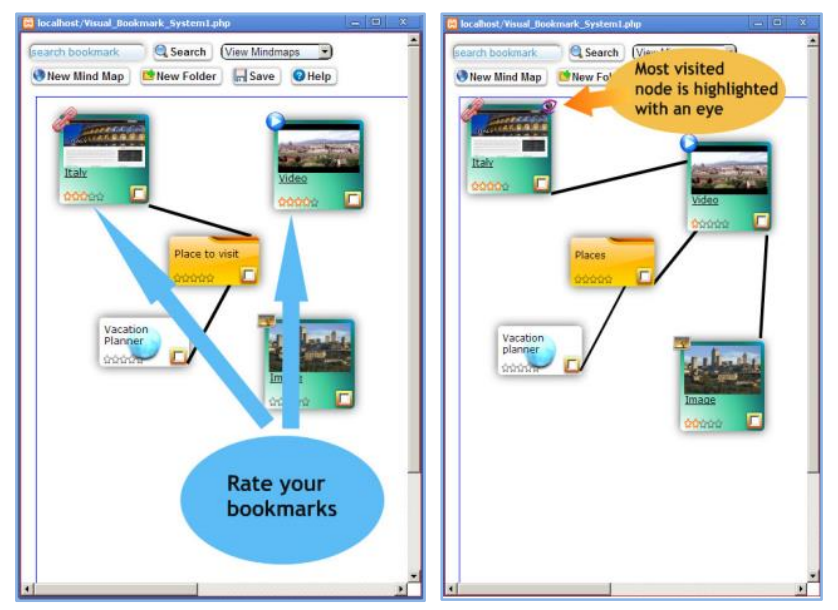

Fig. 7. Five star rating and highligting most visited bookmarks

Afterwards, when user in need of looking back into previously searched information, they can search within this new system using keyword just like searching using web search engines, and then the system will respond with boxes filled up with tags that matched with respective keywords, where each box represents a mind map. User can browse and edit the mind maps by clicking on the respective tag boxes [Figure 8].

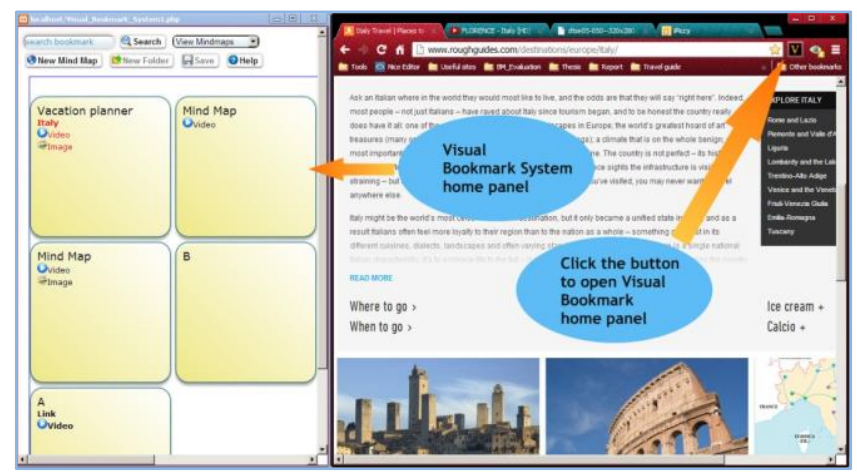

Fig. 8. All mind maps with bookmark tags

Finally, the features of the proposed system help users to organize their bookmarks in such a way that users are able to find back easily their previously searched websites' most expected information within their browser that will minimize the probabilities of searching them again with the help of search engines or re-visiting them.

\section{Evaluation}

In order to test whether people have difficulty with the concept of proposed system, a short preliminary study has been conducted in which 15 people were requested to use the first prototype of the VisMarkMap as a bookmarking system. Here, the people who were interviewed are students and teachers of school and universities. The experiment is conducted in two phases. In the first phase, initially, users were introduced briefly about the VisMarkMap System. Then users were given a specific scenario [Figure 9] to perform some searching and bookmarking both with the traditional bookmark systems (i.e. chrome browser bookmark, Delicious, Pinterest) as well as with this new visual bookmark system (VisMarkMap).

The initial goal of the first phase was to measure the performance of the users on using the user interface of the first prototype of the new visual bookmark system. In this phase the usability of the user interfaces of the new system is also verified. The test is designed using the format used for formative evaluation by [19]. For measuring the performance, some significant quantifiable usability goals have been chosen [Table 1]. While performing the scenario using the new visual bookmark System, users were directly observed to check how many attempts they exactly need to perform a specific task.

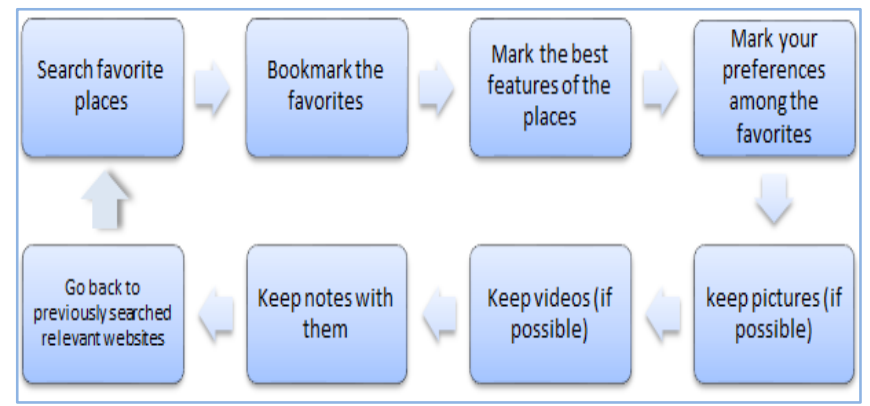

Fig. 9. Vacation Planner Scenario 
TABLE I. USABILITY GOALS TO MEASURE

\begin{tabular}{|l|l|l|l|l|l|}
\hline $\begin{array}{l}\text { Usability } \\
\text { Attribut } \\
\text { e }\end{array}$ & $\begin{array}{l}\text { Measuring } \\
\text { Instrumen } \\
\text { t }\end{array}$ & $\begin{array}{l}\text { Value to } \\
\text { be } \\
\text { Measure } \\
\text { d }\end{array}$ & $\begin{array}{l}\text { Worst } \\
\text { Acceptabl } \\
\text { e Level }\end{array}$ & $\begin{array}{l}\text { Planne } \\
\text { d } \\
\text { Target } \\
\text { Level }\end{array}$ & $\begin{array}{l}\text { Best } \\
\text { Possibl } \\
\text { e Level }\end{array}$ \\
\hline Initial use & $\begin{array}{l}\text { Create a } \\
\text { new mind } \\
\text { map }\end{array}$ & $\begin{array}{l}\text { Number } \\
\text { of attempt }\end{array}$ & 3 & 2 & 1 \\
\hline Initial use & $\begin{array}{l}\text { Create a } \\
\text { custom } \\
\text { node }\end{array}$ & $\begin{array}{l}\text { Number } \\
\text { of attempt }\end{array}$ & 3 & 2 & 1 \\
\hline Initial use & $\begin{array}{l}\text { Create a } \\
\text { bookmark }\end{array}$ & $\begin{array}{l}\text { Number } \\
\text { of attempt }\end{array}$ & 3 & 2 & 1 \\
\hline Initial use & $\begin{array}{l}\text { Connect the } \\
\text { nodes in a } \\
\text { mind map }\end{array}$ & $\begin{array}{l}\text { Number } \\
\text { of attempt }\end{array}$ & 3 & 2 & 1 \\
\hline Initial use & $\begin{array}{l}\text { Tagging the } \\
\text { nodes }\end{array}$ & $\begin{array}{l}\text { Number } \\
\text { of attempt }\end{array}$ & 3 & 2 & 1 \\
\hline Initial use & $\begin{array}{l}\text { Preview the } \\
\text { nodes }\end{array}$ & $\begin{array}{l}\text { Number } \\
\text { of attempt }\end{array}$ & 3 & 2 & 1 \\
\hline Initial use & $\begin{array}{l}\text { Delete a } \\
\text { connection } \\
\text { between } \\
\text { nodes }\end{array}$ & $\begin{array}{l}\text { Number } \\
\text { of attempt }\end{array}$ & 3 & 2 & 1 \\
\hline
\end{tabular}

In the second phase, users were requested to perform the same scenario again after couple of days. During the second phase users are again observed to find out the tendency of using search engines to find out the previously searched websites. After the completion of the second phase, questionnaire method was used to measure the usability of the new system against the all other well-known bookmarking systems such as traditional browser bookmark, Delicious and Pinterest. The questionnaire was designed carefully using several standard software usability measuring questionnaire $[20,21,22]$, to measure the four significant areas of the usability such as,

- Usefulness and effectiveness

- Ease of use

- Ease of learning

- Satisfaction

The questionnaire was built based upon the Likert scale and the respondents were allowed to indicate their agreement or disagreement with a 7 point scale.

\section{A. Evaluation Results}

a) First Phase

In Figure 10 to Figure 16, the outcome of the user observation during the first phase is presented. It is found, in most cases majority of users were able to finish the various tasks on their first trial, without any mistake, whereas, in some cases, one third of the users made at most one mistake and were able to finish the tasks at most with two trials.

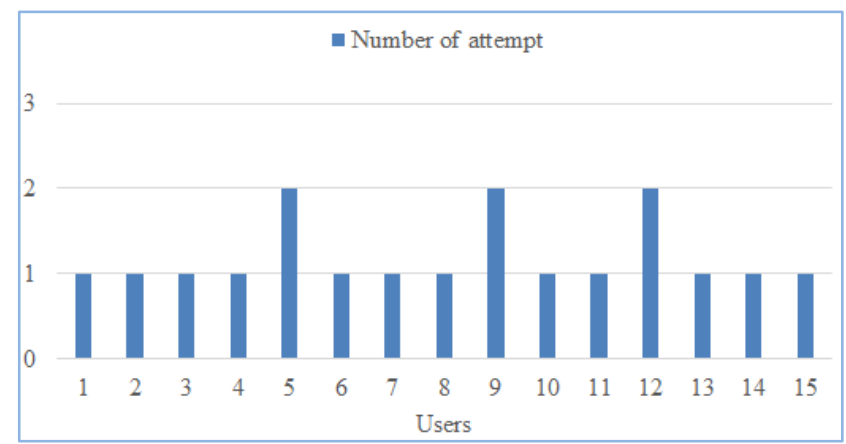

Fig. 10. Create a new mind map

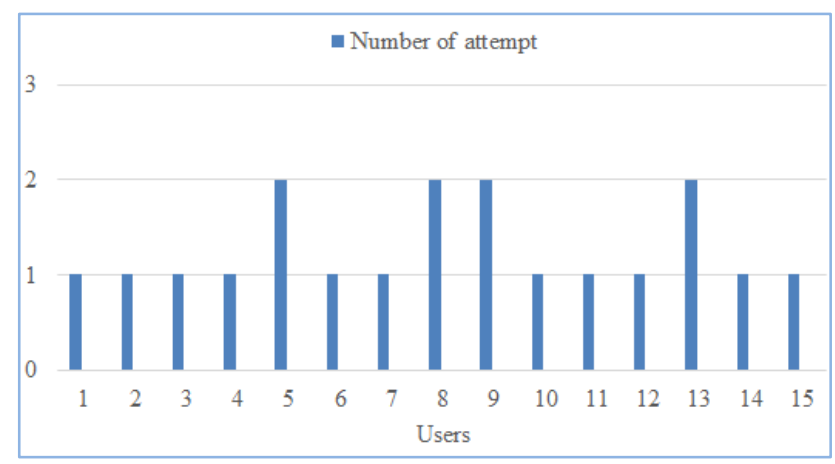

Fig. 11. Create a custom node

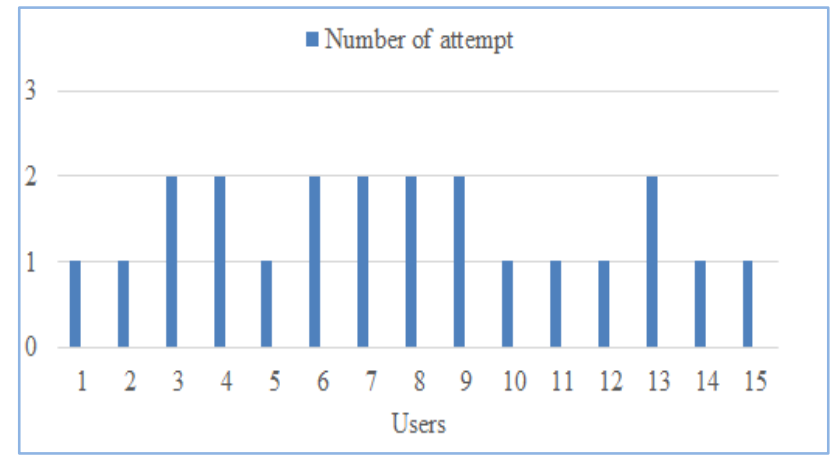

Fig. 12. Create a bookmark

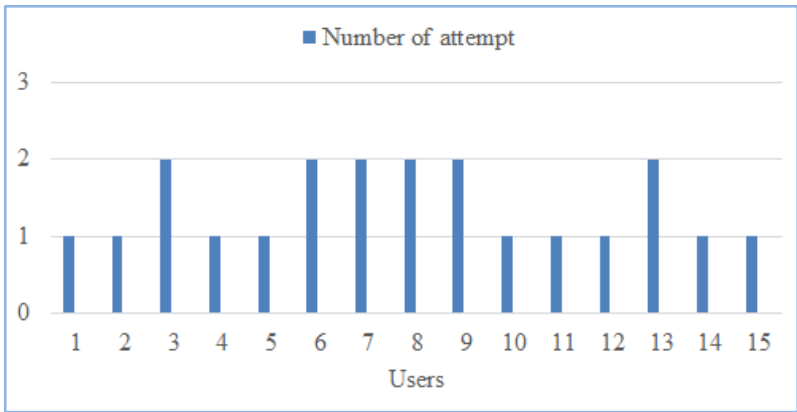

Fig. 13. Connect the bookmark nodes 


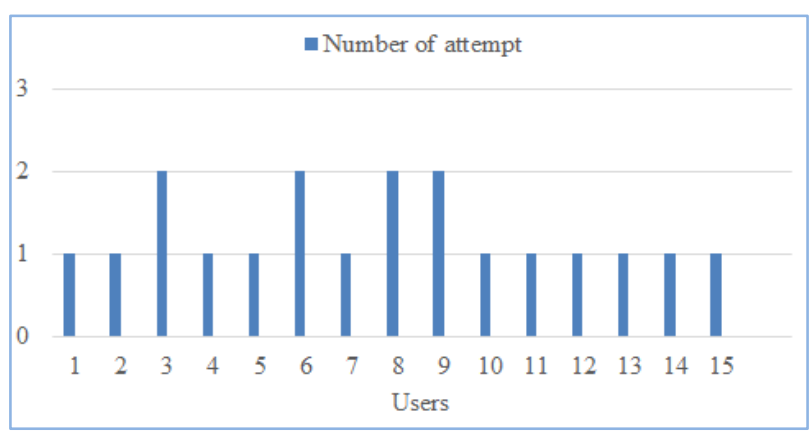

Fig. 14. Tagging the nodes

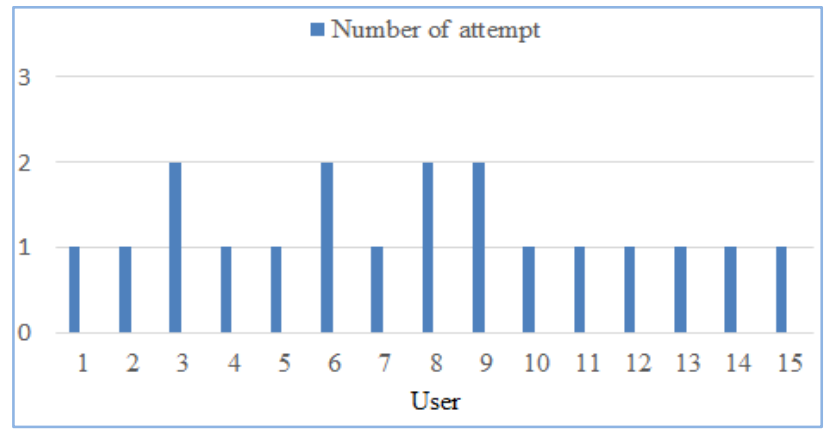

Fig. 15. Preview the bookmarks

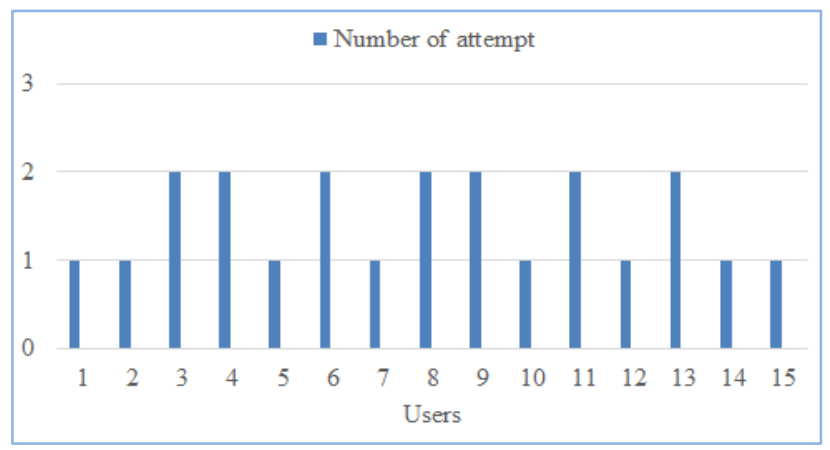

Fig. 16. Removing a connection between nodes

\section{b) Second phase}

During the second phase of user observation it has been recorded how many times a particular user attempt for search engines while using the existing bookmark systems and the new visual bookmarking system, to find out previously searched websites' information. The experiments results for Delicious, Pinterest and new system are shown on Figure 17, Figure 18 and Figure 19 respectively.

Figure 19 shows more than $50 \%$ users ( 8 out of 15 ) did not attempt for search engines at all while to find out previously searched websites' information after understanding the features provided by the new system about which they came to know during the first phase, which helps users by minimizing their effort in case of comparing and finding the desired websites from search results returned as a list by the search engines. Using the new bookmark system, the percentage of people who attempted for search engines is reasonably lower compared to the other systems. It was also observed, most of the time almost all of the users attempted for search engines while they were using chrome browser bookmark system.

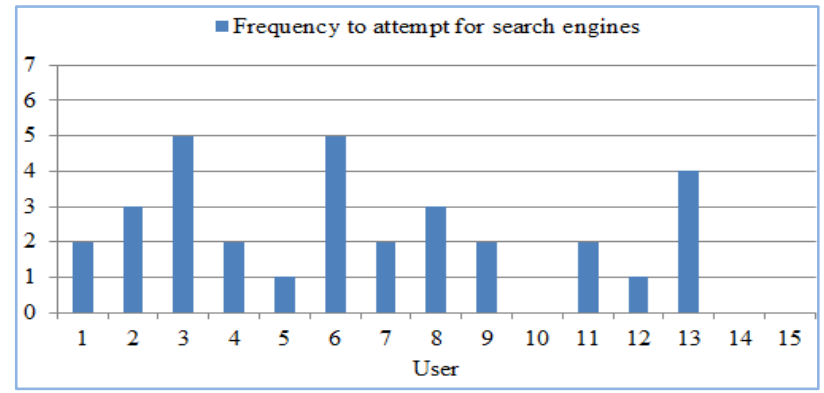

Fig. 17. Frequency to attempt for search engines using Delicious

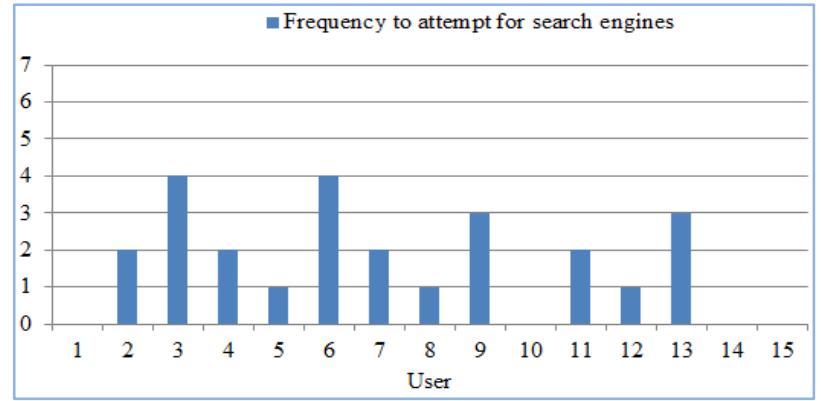

Fig. 18. Frequency to attempt for search engines using Pinterest

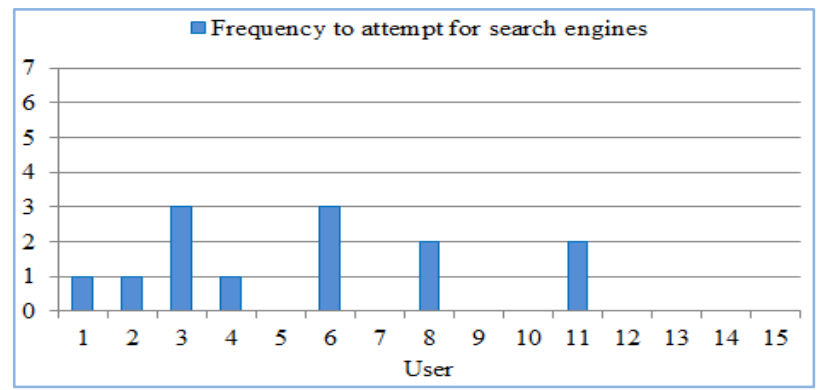

Fig. 19. Frequency to attempt for search engines using the new system

From the collected responses from questionnaire phase it has been found that most people agreed upon the fact that with the VisMarkMap they are able to bookmark according to their expectation (60\% agreed \& 20\% strongly agreed) [Figure 20]. Significant numbers of people think VisMarkMap is making them more productive (30\% strongly agreed \& $40 \%$ agreed) [Figure 20] and most people think it as more useful (40\% strongly agreed and $40 \%$ agreed) than the other bookmarking systems [Figure 20].

A key number of users found the VisMarkMap is easy to use $(40 \%$ strongly agreed, $10 \%$ agreed \& $20 \%$ agreed more than disagree) and easy to learn (50\% strongly agreed \& $40 \%$ agreed) [Figure 21]. Most importantly, majority of people (40\% agreed \& 30\% strongly agreed) were satisfied about the overall functionalities of the first prototype of the new visual bookmarking system (VisMarkMap) [Figure 22]. 


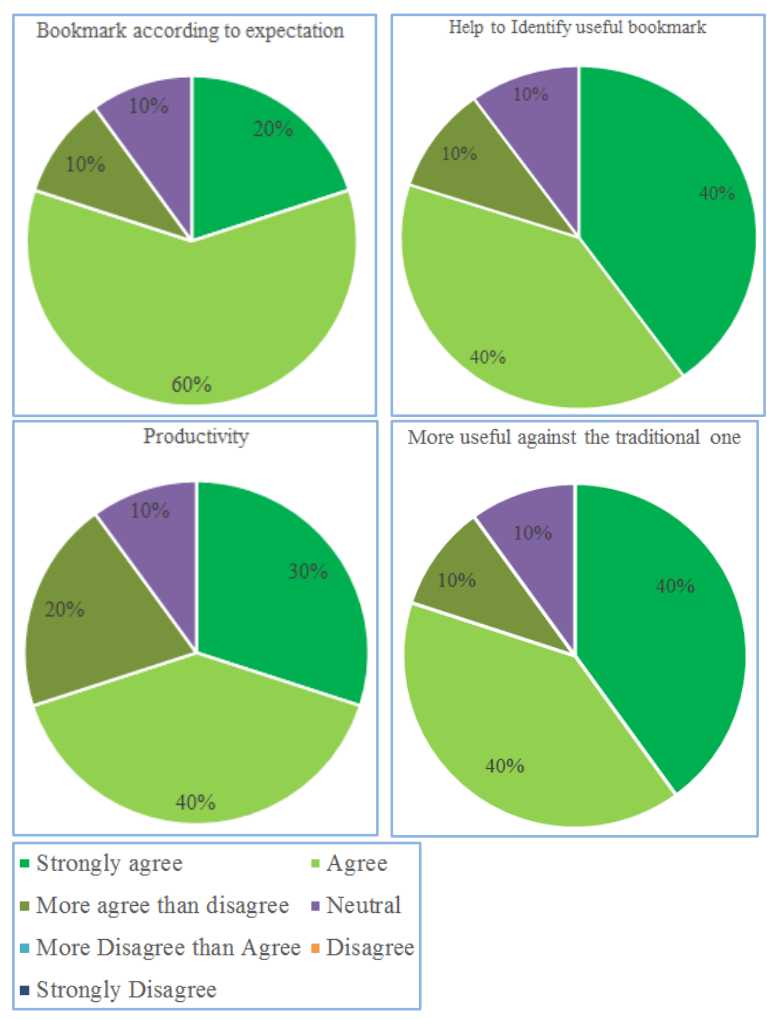

Fig. 20. Usefulness of VisMarkMap

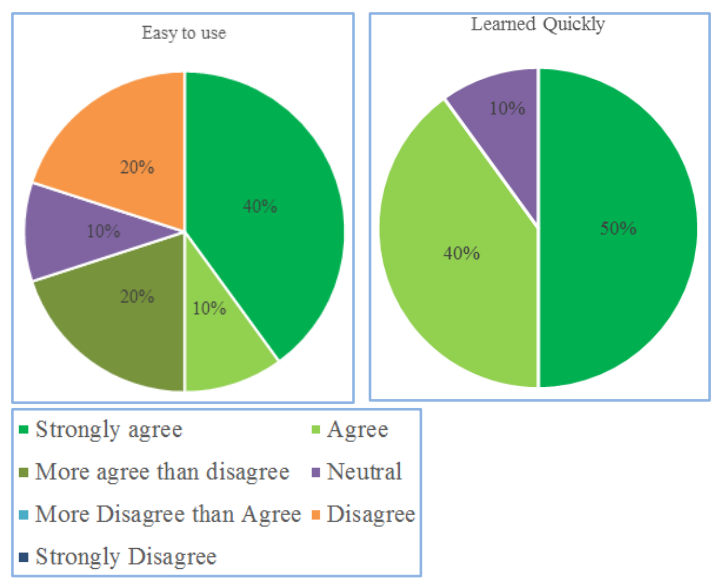

Fig. 21. Ease of use of the VisMarkMap

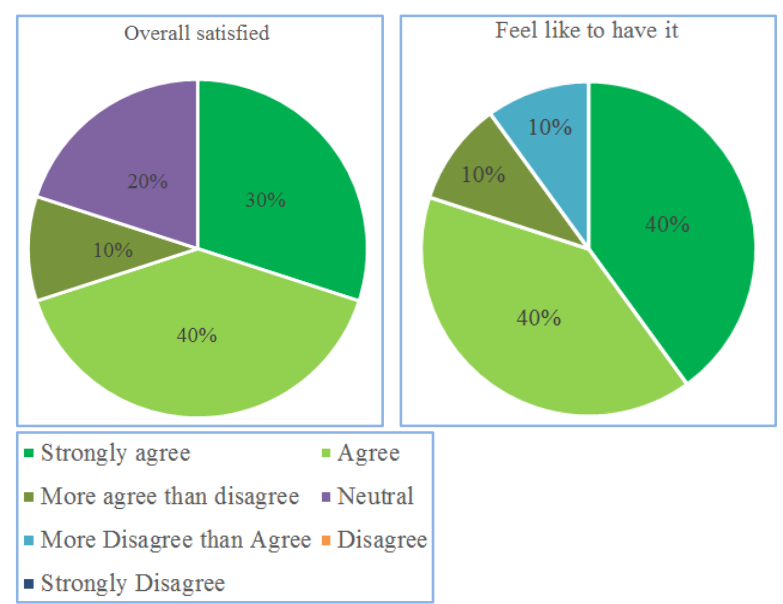

Fig. 22. Satisfaction of VisMarkMap

\section{DISCUSSION}

The preliminary observations suggest that VisMarkMap considered as most useful to the major number of users as they were able to do all the desired tasks regarding bookmarking with more organized way in a mind map. The best aspects of this new bookmarking system are mentioned by the users are:

- The visual representation with mind map makes easy the process of remembering the correlations among different bookmarks and causes less mental effort to find out the expected ones. In addition, the five star rating helps them to take quick decision in choosing the most important bookmark.

- The personal and the web notes taking facilities and viewing them along with the zoomed preview give them more flexibility to recall about a bookmark than any other available bookmarking systems.

\section{CONCLUSION AND FUTURE WORK}

The goal of this work is to find out a new visual bookmarking technique for letting users to organize bookmarks using mind map along with the most expected information, which will help the users to recall easily the previously searched websites' information from the bookmarks and will also minimize the tendency to revisit or research the website using the search engines. 
For the future work, the present prototype can be improved further to enhance the scalability and usability. In addition, more experiments with this new system are planned to conduct with more users in the field studies to test with more real time scenarios.

\section{REFERENCES}

[1] Zhang,Ping \& Galleta,Dennis (2006). Human Computer Interaction and Management Information Systems Foundations.

[2] Alpert,S.R.,\& Gruenenberg,K.(2000). Concept mapping with multimedia on the web. Jour-nal of Educational Multimedia and Hypermedia, 9(4), 313-330.

[3] Peter Mika (2007).Social Networks and the Semantic Web, Semantic Web and Beyond.

[4] LjupcoJovanoski, Vladimir Apostolski and DimitirTrajanov (2010). Comparing Social Bookmarking and Tagging Systems: Towards Semantic Sharing Platforms.

[5] Kaasten,Shaun (2000) \&Greenberg,Saul. Designing an Integrated Bookmark / History System for Web Browsing. In Proceedings of the Western Computer Graphics Symposium 2000, (Panorama Mountain Village, BC, Canada), March 26-29, 2000

[6] Cañas, A.J., Leake, D.B., \& Wilson, D.C. (1999). Managing, mapping and manipulating con-ceptual knowledge. AAAI Workshop Technical Report WS-99-10: Exploring the synergies of knowledge management $\&$ case-based reasoning. Menlo Park, CA: AAAI Press.

[7] John W. Budd (2004). Mind Maps as Classroom Exercises. The Journal of Economic Education, Vol. 35, No. 1 (Winter, 2004), pp. 35-46 Published by: Taylor \& Francis, Ltd. Article Stable.

[8] Tanja Keller and Sigmar-Olaf Tergan,Visualizing Knowledge and Information: An Introduction Lecture Notes in Computer Science Volume 3426, 2005, pp 1-23.

[9] Gotz, D. The ScratchPad: sensemaking support for the web. In Proc. Of WWW 2007, 1329-1330.

[10] Levene, M. (2006). An Introduction to Search Engines and Web Navigation. Reading, MA: Addison-Wesley
[11] Tauscher, L. and Greenberg, S., How People Revisit Web Pages: Empirical Findings and Implications for the Design of History Systems. In International Journal of Human-Computer Studies, pages 47(1), 97138, 1997.

[12] Abrams, D., Baecker, R. and Chignell, M. Information Archiving with Bookmarks: Personal Web Space Construction and Organization. In Proceedings of the ACM/SIGCHI Conference on Human Factors in Computing Systems (CHI'98), pages 18-23, 1998.

[13] Cernea, D., Ebert, A., Kerren, A., \& Truderung, I.(2013). WebComets: A Tab-Oriented Approach for Browser History Visualization. GRAPP.

[14] Sweller, J., \& Chandler, P. (1994). Why some material is difficult to learn. Cognition and In-struction, 12 (3), 185-233.

[15] Cox,R.(1999).Representation, construction, externalised cognition and individual differences. Learning and Instruction, 9, 343-363.

[16] Larkin,J.H. (1989).Display-based problem solving. In D. Klahr, \& K. Kotovsky (Eds.), Complex information processing. The impact of Heribert Simon(pp. 319-342). Hillsdale, NJ: Lawrence Erlbaum Associates.

[17] Larkin,J.H., \& Simon, H.A. (1987).Why a diagram is (sometimes) worth 10.000 words. Cognitive Science, 11, 65-100.

[18] Scaife,M., \& Rogers, Y. (1996). External cognition: how do graphical representations work? Int. J. Human-Computer Studies, 45, 185-213.

[19] Hix,Deborah and Hartson,H.Rex (1992). Formative Evaluation: Ensuring Usability in User Interfaces. Technical Report TR-92-60, Computer Science, Virginia Polytechnic Institute and State University.

[20] Lund,A.M.(2001).Measuring usability with the USE questionnaire. Usability and User Experience, 8(2), 8

[21] Lewis,J.R.(1995).IBM computer usability satisfaction questionnaires: psychometric evaluation and instructions for use. International Journal of Human-Computer Interaction, 7(1), 57-78.

[22] Brooke,John(1996). SUS-A quick and dirty usability scale. Usability evaluation in industry, 189, 194. 